\title{
Statistical seismology of transverse waves in the solar corona
}

\author{
E. Verwichte ${ }^{1,2}$, T. Van Doorsselaere ${ }^{2}$, R. S. White ${ }^{1}$, and P. Antolin ${ }^{2}$ \\ 1 Department of Physics, University of Warwick, Coventry CV4 7AL, UK \\ e-mail: Erwin.Verwichte@warwick.ac.uk \\ 2 Centre for Plasma Astrophysics, Department of Mathematics, Katholieke Universiteit Leuven, Celestijnenlaan 200B, 3001 Leuven, \\ Belgium
}

Received 27 September 2012 / Accepted 27 February 2013

\begin{abstract}
Context. Observations show that transverse oscillations commonly occur in solar coronal loops. The rapid damping of these waves has been attributed to resonant absorption. The oscillation characteristics carries information of the structuring of the corona. However, self-consistent seismological methods that extract information from individual oscillations are limited because there are fewer observables than unknown parameters in the model, and the problem is underdetermined. Furthermore, it has been shown that one-to-one comparisons of the observed scaling of period and damping times with wave damping theories are misleading.

Aims. We aim to investigate whether seismological information can be gained from the observed scaling laws in a statistical sense. Methods. A statistical approach is used whereby scaling laws are produced by forward modelling using distributions of values for key loop cross-sectional structuring parameters. We study two types of observations: 1) transverse loops oscillations as seen mainly with TRACE and SDO and 2) running transverse waves seen with the Coronal Multichannel Polarimeter (CoMP).

Results. We demonstrate that the observed period-damping time scaling law does provide information about the physical damping mechanism, if observations are collected from as wide range of periods as possible and a comparison with theory is performed in a statistical sense. The distribution of the ratio of damping time over period, i.e. the quality factor, has been derived analytically and fitted to the observations. A minimum value for the quality factor of 0.65 has been found. From this, a constraint linking the ranges of possible values for the density contrast and inhomogeneity layer thickness is obtained for transverse loop oscillations. If the layer thickness is not constrained, then the density contrast is at most equal to 3. For transverse waves seen by CoMP, it is found that the ratio of maximum to minimum values for these two parameters has to be less than 2.06; i.e., the sampled values for the layer thickness and Alfvén travel time come from a relatively narrow distribution.

Conclusions. Now that more and more transverse loop oscillations have been analysed, a statistical approach to coronal seismology becomes possible. Using the observed data cloud, we have found restrictions to the loop's density contrast and inhomogeneity layer thickness. Surprisingly, for running waves, narrow distributions for loop parameters have been found.
\end{abstract}

Key words. Sun: oscillations - magnetohydrodynamics (MHD)

\section{Introduction}

Transverse waves are pervasive in the solar corona. They have been detected with confidence ever since 1998 (Aschwanden et al. 1999; Nakariakov et al. 1999) in the form of transverse loop oscillations (TLOs). To date, more than 50 TLOs have been reported with periods ranging between $100 \mathrm{~s}$ and $3 \mathrm{~h}$ (Aschwanden et al. 2002; Wang \& Solanki 2004; Verwichte et al. 2004; Hori et al. 2005, 2007; Van Doorsselaere et al. 2007, 2009; De Moortel \& Brady 2007; Verwichte et al. 2009, 2010; Mrozek 2011; White \& Verwichte 2012; White et al. 2012; Verwichte et al. 2012). The majority of these oscillations have been studied using EUV imagers, such as TRACE (Handy et al. 1999), EUVI/STEREO (Howard et al. 2008) and AIA/SDO (Lemen et al. 2012). They are reported to dampen quickly with oscillation quality factors in the range 0.6-5.4.

Tomczyk et al. (2007) used ground-based spectral measurements with the Coronal Multichannel Polarimeter (CoMP) (Tomczyk et al. 2008) to demonstrate that small-amplitude propagating transverse waves are ubiquitous in the solar corona. This result seems to be supported by the recent report of running transverse waves in coronal loops by AIA/SDO (McIntosh et al. 2011; Wang et al. 2012).

A widely accepted explanation for the rapid damping is the mechanism of resonant absorption where the transverse wave is considered to be an Alfvénic kink mode (or surface Alfvén mode Wentzel 1979; Goossens et al. 2012) whose nature evolves through a resonance at a loop layer where its frequency matches the local Alfvén frequency, from a global transverse loop motion to a local, mainly azimuthal motion (Ruderman \& Roberts 2002; Goossens et al. 2002). Once local, the mode cannot be observed directly and it then proceeds to dampen dissipatively, enhanced by phase-mixing (or alternatively collisionlessly). It is the rate of mode evolution from global to local that is observed as the rapid damping of the transverse wave. The observed damping time depends on the structure of the Alfvén frequency across the loop.

Besides the loop's average Alfvén speed and magnetic field strength (Nakariakov \& Ofman 2001), there is the potential for seismologically determining the loop cross-section profile, including the density contrast, which is difficult to measure directly (e.g. Aschwanden et al. 2003; Schmelz et al. 2003; Terzo $\&$ Reale 2010). By combining the theories for the propagation and damping of the transverse wave it is possible to constrain the unknown parameters in the problem (Verwichte et al. 2006) self-consistently. However, for the resonant absorption damping model, the problem is under-determined and it is not possible to deduce both density contrast and the thickness of the inhomogeneity layer independently (Arregui et al. 2007; Goossens et al. 2008; Arregui \& Asensio Ramos 2011). 
Ofman \& Aschwanden (2002) modelled the scaling relations, e.g. between damping time and period, for different damping mechanisms. They find that the observed scaling relations were more compatible with phase mixing. However, it is pointed out by Arregui et al. (2008) that a one-to-one comparison between the observed scaling and the linear scaling from resonant absorption inherently makes the unrealistic assumption that all loops have the same cross-sectional structuring. In fact, by allowing the cross-sectional profile to vary between events, they show that the scaling from resonant absorption can easily depart from linear. Thus, they conclude that scaling laws were not sufficient to distinguish damping mechanisms, because resonant absorption can reproduce several dependencies using carefully chosen distributions of equilibrium parameters. However, now it becomes possible to use the inverse approach. Since 2002 (Aschwanden et al. 2002), the number of observations and the range of observed periods has increased. Given the observed scaling laws of periods and damping time, can we find information on the statistical distributions of equilibrium parameters of coronal loops that exist in the solar corona? In this article we show that it is possible to use statistical and forward-modelling approaches to model scaling laws of loops. This statistical, seismological information on the coronal loop ensemble can potentially help distinguish between different coronal loop models and heating mechanisms.

The paper is structured in two main parts. Section 2 statistically investigates the scaling of TLOs using two approaches. Section 3 studies the transverse waves seen by CoMP (Tomczyk et al. 2007) statistically. We discuss our findings in Sect. 4.

\section{Statistics of transverse loop oscillations}

Since 2002, when Ofman \& Aschwanden (2002) modelled the scaling relations for standing TLOs, many more observations have been analysed. Table 1 lists 52 events of TLOs from 13 studies. Figure 1 shows the distribution of damping times, $\tau$, versus oscillation period, $P$. We can find a power-law relationship between those two observed quantities as

$\tau=\alpha P^{\gamma}, \log _{10} \alpha=0.44 \pm 0.31, \gamma=0.94 \pm 0.12$.

Under the assumption of a loop where the density drops from inner to external conditions over a thin transition layer, the resonant absorption rate is given by (e.g. Ionson 1978; Hollweg \& Yang 1988; Goossens et al. 1992; Ruderman \& Roberts 2002)

$\tau=\xi_{\mathrm{E}} P, \quad \xi_{\mathrm{E}}(\ell / a, \zeta)=F(\ell / a)^{-1} \frac{\zeta+1}{\zeta-1}$,

where $F, \ell, a$ and $\zeta$ are parameters that describe the crosssectional profile of the loop mass density, $\rho(r)$. Here, we choose a half-wavelength sinusoidally varying transition layer, i.e.

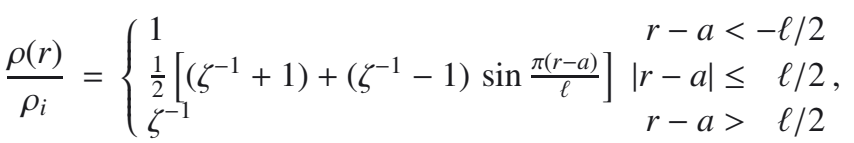

where $\rho_{i}$ is the loop-axis equilibrium density and $\zeta$ the ratio of the loop-axis density over the external density, i.e. $\zeta=\rho_{i} / \rho_{e}$. For such a profile, $F=2 / \pi$. Equation (2) is, strictly speaking, only valid in the regime where $\ell \ll a$, though Van Doorsselaere et al. (2004) shows that it still provides a relatively accurate extension into the regime of finite resonance layer widths. Also,
Table 1. Characteristics of observed TLOs.

\begin{tabular}{|c|c|c|c|c|}
\hline$\#$ & $P(s)$ & $\tau(s)$ & $L(M m)$ & Reference $^{1}$ \\
\hline 1 & 261 & 870 & 168 & $\mathrm{~A} 02$ \\
\hline 2 & 265 & 300 & 72 & \\
\hline 3 & 316 & 500 & 174 & \\
\hline 4 & 277 & 400 & 204 & \\
\hline 5 & 272 & 849 & 162 & \\
\hline 6 & 435 & 600 & 258 & \\
\hline 7 & 143 & 200 & 166 & \\
\hline 8 & 423 & 800 & 406 & \\
\hline 9 & 185 & 200 & 192 & \\
\hline 10 & 396 & 400 & 146 & \\
\hline 11 & 234 & 714 & $350 \pm 50$ & WS04 \\
\hline 12 & $249 \pm 33$ & $920 \pm 360$ & 218 & V04 \\
\hline 13 & $448 \pm 18$ & $1260 \pm 500$ & 218 & \\
\hline 14 & $392 \pm 31$ & $1830 \pm 790$ & 228 & \\
\hline 15 & $382 \pm 12$ & $1330 \pm 528$ & 233 & \\
\hline 16 & $358 \pm 30$ & $1030 \pm 570$ & 237 & \\
\hline 17 & $326 \pm 45$ & $980 \pm 400$ & 235 & \\
\hline 18 & $357 \pm 89$ & $1320 \pm 720$ & 236 & \\
\hline 19 & 567 & 1500 & $400 \pm 100$ & H05 \& H07 \\
\hline 20 & 918 & 4200 & $800 \pm 200$ & \\
\hline 21 & 425 & 2300 & 384 & VD07 \\
\hline 22 & $436 \pm 4.5$ & $2129 \pm 280$ & $400 \pm 40$ & \\
\hline 23 & $243 \pm 6.4$ & 1200 & $400 \pm 40$ & \\
\hline 24 & $895 \pm 2$ & $521 \pm 8$ & 228 & DMB07 \& VD09 \\
\hline 25 & $452 \pm 1$ & $473 \pm 6$ & 228 & \\
\hline 26 & $630 \pm 30$ & $1000 \pm 300$ & $340 \pm 15$ & V09 \\
\hline 27 & $2418 \pm 5$ & $3660 \pm 80$ & $680 \pm 50$ & V10 \\
\hline 28 & 377 & 500 & 250 & M11 \\
\hline 29 & $225 \pm 40$ & $240 \pm 45$ & $121 \pm 12$ & WV12 \\
\hline 30 & $215 \pm 5$ & $293 \pm 18$ & $111 \pm 11$ & \\
\hline 31 & $213 \pm 9$ & $251 \pm 36$ & $132 \pm 13$ & \\
\hline 32 & $216 \pm 27$ & $230 \pm 23$ & $113 \pm 11$ & \\
\hline 33 & $520 \pm 5$ & $735 \pm 53$ & $396 \pm 40$ & \\
\hline 34 & $596 \pm 50$ & $771 \pm 336$ & $374 \pm 37$ & \\
\hline 35 & $212 \pm 20$ & $298 \pm 30$ & $279 \pm 28$ & \\
\hline 36 & $256 \pm 22$ & $444 \pm 105$ & $240 \pm 24$ & \\
\hline 37 & $135 \pm 9$ & $311 \pm 85$ & $241 \pm 24$ & \\
\hline 38 & $115 \pm 2$ & $175 \pm 30$ & $159 \pm 16$ & \\
\hline 39 & $103 \pm 8$ & $242 \pm 114$ & $132 \pm 13$ & \\
\hline 40 & $302 \pm 14$ & $306 \pm 43$ & $466 \pm 50$ & W12 \\
\hline 41 & $565 \pm 4$ & $666 \pm 42$ & $301 \pm 30$ & V12 \\
\hline 42 & $222 \pm 18$ & $420 \pm 360$ & $274 \pm 30$ & \\
\hline 43 & $474 \pm 12$ & $900 \pm 120$ & $400 \pm 30$ & \\
\hline 44 & $1170 \pm 6$ & $1218 \pm 48$ & $400 \pm 30$ & \\
\hline 45 & $623 \pm 4$ & $960 \pm 60$ & $270 \pm 30$ & \\
\hline 46 & $150 \pm 5$ & $216 \pm 60$ & $188 \pm 20$ & \\
\hline 47 & $122 \pm 6$ & $348 \pm 360$ & $160 \pm 20$ & \\
\hline 48 & $273 \pm 54$ & $468 \pm 36$ & $171 \pm 20$ & \\
\hline 49 & $282 \pm 6$ & $606 \pm 186$ & $122 \pm 20$ & \\
\hline 50 & $491 \pm 18$ & $834 \pm 6$ & $262 \pm 20$ & \\
\hline 51 & $348 \pm 7$ & $906 \pm 288$ & $238 \pm 20$ & \\
\hline 52 & $340 \pm 3$ & $930 \pm 144$ & $200 \pm 20$ & \\
\hline
\end{tabular}

Notes. ${ }^{(1)}$ The reference citations are listed in Fig. 1.

Eq. (2) does not describe any transient behaviour in the damping (Pascoe et al. 2012).

Equation (2) shows that the resonant absorption time-scale scales linearly with period. This matches the observed scaling well. However, as pointed out by Arregui et al. (2008), a oneto-one comparison is problematic because Eq. (2) also depends 


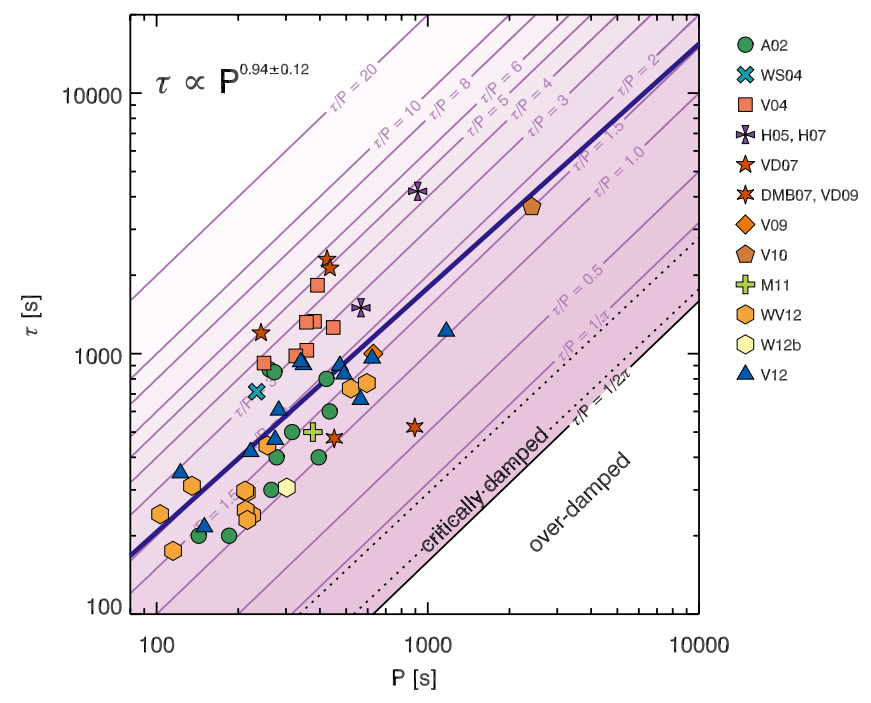

Fig. 1. Damping time versus period of measured TLOs. The thick line is a power-law fit of the form $\tau=\alpha P^{\gamma}$. The parallel lines indicate contours of quality factor $\tau / P$. The symbols correspond to the following publications reporting TLOs. A02: Aschwanden et al. (2002); WS04: Wang \& Solanki (2004); V04: Verwichte et al. (2004); H05: Hori et al. (2005); H07: Hori et al. (2007); VD07: Van Doorsselaere et al. (2007); DMB07: De Moortel \& Brady (2007); VD09: Van Doorsselaere et al. (2009); V09: Verwichte et al. (2009), V10: Verwichte et al. (2010); M11: Mrozek (2011); WV12: White \& Verwichte (2012); W12: White et al. (2012); V12: Verwichte et al. (2012).

on $\ell / a$ and $\zeta$, which will vary between loops. We can identify the observed fit parameter $\alpha$ with $\xi_{\mathrm{E}}(\ell / a, \zeta)$. What possible range of values of $\ell / a$ and $\zeta$ gives the best match between $\alpha$ and $\xi_{\mathrm{E}}$ ?

\subsection{Modelling the damping time-period scaling}

To compare theoretical and observed scaling, the following forward-modelling procedure is adopted. The hidden variables are allowed to have a distribution of plausible values and are assumed to be independent. The distribution of the thickness of the inhomogeneity layer, $\ell / a$, and the density contrast, $\zeta$, are modelled as

$$
\begin{aligned}
\frac{\mathrm{d}(\ell / a)}{\mathrm{d} N} & =H\left(\ell / a,(\ell / a)_{\min },(\ell / a)_{\max }\right), \\
\frac{\mathrm{d} \zeta}{\mathrm{d} N} & =H\left(\zeta, \zeta_{\min }, \zeta_{\max }\right),
\end{aligned}
$$

where $H\left(x, x_{\min }, x_{\max }\right)$ is the top-hat function defined as

$$
H\left(x, x_{\min }, x_{\max }\right)=\left\{\begin{array}{lr}
\left(x_{\max }-x_{\min }\right)^{-1} & x_{\min } \leq x \leq x_{\max } \\
0 & x<x_{\min } \text { or } x>x_{\max }
\end{array}\right.
$$

Alternatively, for $\zeta$, we may also use a Jeffrey's probability density function, $J(x)$, which is defined as

$J\left(x, x_{\min }, x_{\max }\right)=\left[x \ln \left(\frac{x_{\max }}{x_{\min }}\right)\right]^{-1}$.

Inherently, the distributions do not depend on other physical parameters or on the period. Thus, we make the assumption that the distribution of these parameters is the same for all sizes of loops. Also, the oscillation period has a distribution

$\frac{\mathrm{d}_{\log _{10} P}}{\mathrm{~d} N}=H\left(\log _{10} P, \log _{10} P_{\max }, \log _{10} P_{\min }\right)$,

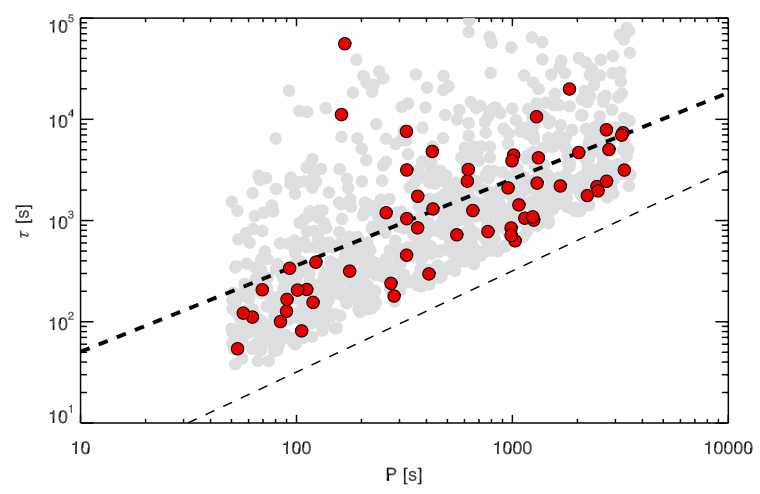

Fig. 2. Example of a realisation of a set of forward-modelled set of $\left(P_{i}, \tau_{i}\right)$ of the same number as currently reported TLOs. The grey circles indicate a realisation of a 1000 sets. Here, $\zeta$ and $\ell / a$ are sampled uniformly from the intervals $[0,4]$ and $[0,2]$, respectively.

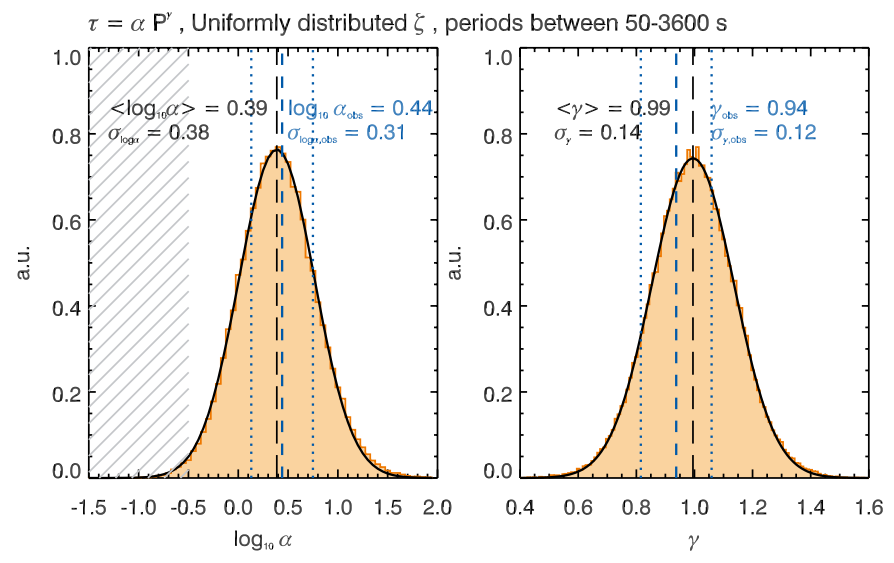

Fig. 3. Forward modelling of the power law for resonant absorption model with uniform distributions. $\ell / a$ and $\zeta$ are sampled uniformly from the intervals $[0,2]$ and $[0,4]$, respectively. The hatched region denoted values of $\alpha$ less than $F / 2$, which Eq. (2) does not permit.

with $P_{\text {min }}=50 \mathrm{~s}$ and $P_{\max }=3600 \mathrm{~s}$, chosen to reflect the bias of observers to identify and study oscillations in the range of several minutes.

There are $M$ number of observations of TLOs. We thus sample $M$ values from these distributions to produce $M$ sets of values $\left((\ell / a)_{i}, \zeta_{i}, P_{i}\right), i \in[1, M]$. The corresponding values of $\tau_{i}$ are calculated using Eq. (2). Figure 2 shows an example of a realisation. Then, a power law such as Eq. (1) is fitted to this realisation of $M$ pairs of values $\left(P_{i}, \tau_{i}\right)$ and $\alpha$ and $\gamma$ determined. This process is repeated to produce $N$ realisations of $\alpha$ and $\gamma$. The distribution of $\alpha$ and $\gamma$, its mean and standard deviation, are then compared with the observations. Figure 3 shows how the forward-modelled distribution of the observed scaling parameters $\alpha$ and $\gamma$ match the observed values well with similar uncertainties. The same conclusions can be drawn when using a Jeffrey's distribution for $\zeta$.

Now we wish to investigate whether, by optimising the fit between forward modelled and observed distribution, we can constrain the intervals of $\zeta$ and $\ell / a$, hence extract seismologically information about the transverse structuring of the oscillating loops. Unfortunately, because the spread of $\alpha$ is large, it is difficult to constrain both hidden variables. Therefore, we decide to fix $\ell / a$ to always be sampled uniformly from the interval $[0,2]$. We also fix $\zeta_{\min }$ to be unity. Thus, the only remaining free parameter is $\zeta_{\max }$. For each value of $\zeta_{\max }$ we compute a forwardmodelled distribution and find its scaling parameters. Figure 4 shows how the forward-modelled value of $\alpha$ varies as a function 

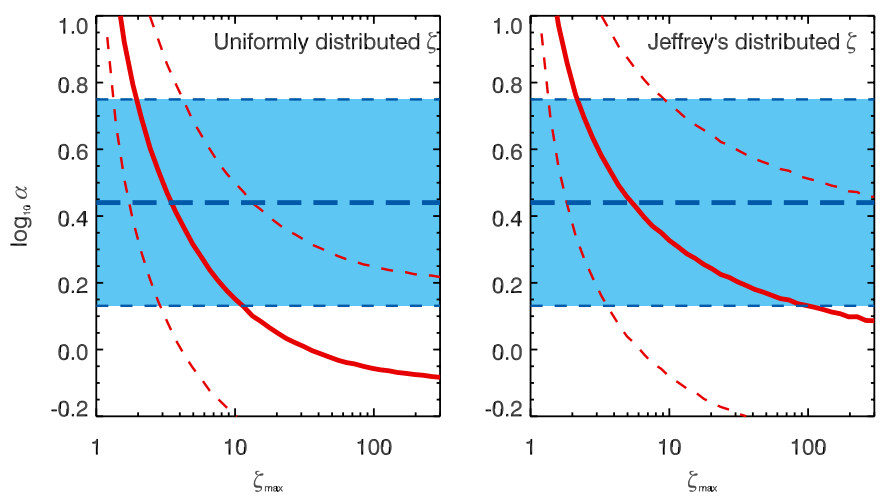

Fig. 4. The mean parameter $\log _{10} \alpha$ as a function of $\zeta_{\max }$ with $\zeta_{\min }=1$ obtained from $N$ fits to a forward modelled sample of values of periods and damping times, using the uniform distribution for $\ell / a$ given in Eq. (4) and a uniform (left) and a Jeffrey's (right) distributions for $\zeta$ as given in Eqs. (4) and (6), respectively. The dashed curves bound the one- $\sigma$ variation in $\log _{10} \alpha$. The horizontal long-dashed line shows the observed value of $\log _{10} \alpha$ bounded by its one- $\sigma$ variation.

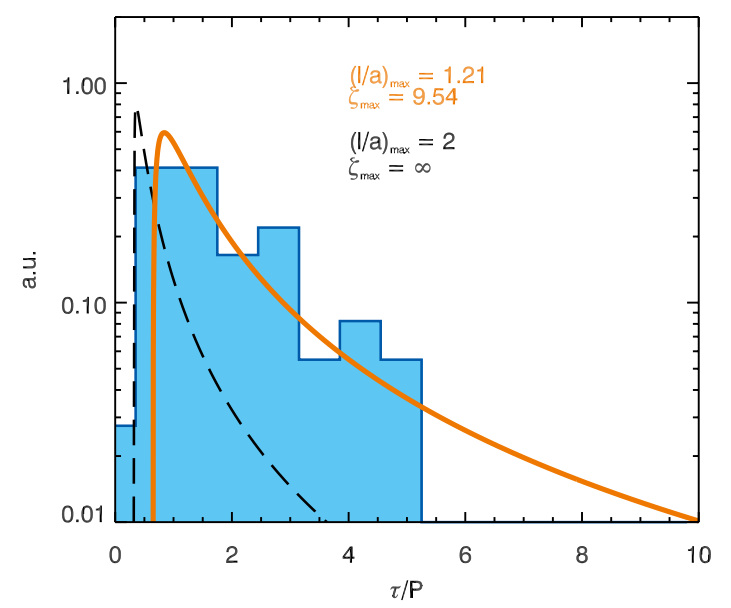

Fig. 5. Distribution of quality factor, $\tau / P$, from the observations (blue distribution) and from the analytical distribution using Eq. (11) with $\ell / a_{\max }=1.2$ and $\zeta_{\max }=9.5$.

of $\zeta_{\max }$. Though the error bars are quite large, we can identify the optimal value $\zeta_{\max }=3$, and suggest that most likely $\zeta$ lies in the range $[1,10]$.

\subsection{The quality-factor probability density distribution}

We use a second method to constrain $\ell / a$ and $\zeta$ by considering the distribution of the quality factor. As Fig. 2 illustrates, a set of values sampled from the distributions in $\ell / a$ and $\zeta$ and using Eq. (2), leads to a non-uniform spread of values in the $P-\tau$ parameter space. We derive this distribution analytically from the distributions of $\ell / a$ and $\zeta$. We make use of the following relations for distributions of dimensionless quantities $x, y$ and $z$ where the relations $y=y(x)$ and $z=x y$ are monotonic:

$\frac{\mathrm{d} y}{\mathrm{~d} N}=\left|\frac{\mathrm{d} x(y)}{\mathrm{d} y}\right| \frac{\mathrm{d} x}{\mathrm{~d} N}, \frac{\mathrm{d} z}{\mathrm{~d} N}=\int_{-\infty}^{+\infty} \frac{\mathrm{d} x}{\mathrm{~d} N} \frac{\mathrm{d}(z / x)}{\mathrm{d} N} \frac{\mathrm{d} x}{|x|}$

We introduce the notations $h(\zeta)=(\zeta+1) /(\zeta-1), y=F(\ell / a)^{-1}$ and $q=\tau / P$ for the inverse Atwood number, inverse inhomogeneity scale length, and quality factor, respectively (The

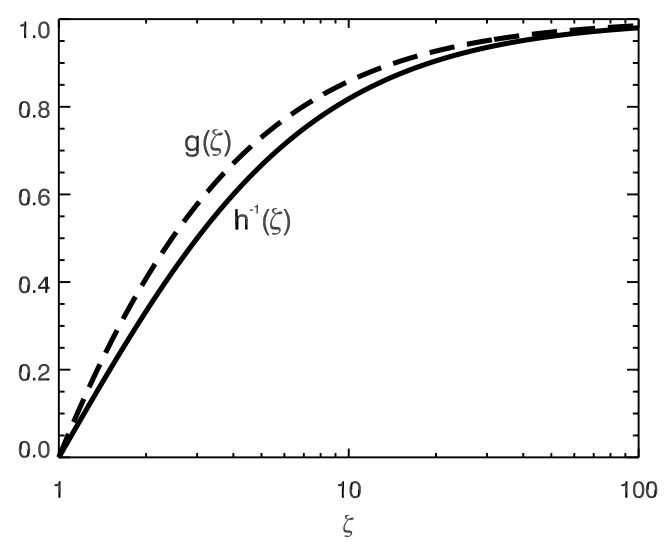

Fig. 6. The dependence of $h^{-1}(\zeta)$ and $g(\zeta)$ on $\zeta$.

function $h(\zeta)$ is shown in Fig. 6). Equation (2) then simply reads as $q=y h$. We find for uniform distributions of $\ell / a$ and $\zeta$

$$
\begin{aligned}
\frac{\mathrm{d} y}{\mathrm{~d} N} & =\frac{1}{y^{2}} H\left(y, F(\ell / a)_{\max }^{-1}, F(\ell / a)_{\min }^{-1}\right), \\
\frac{\mathrm{d} h}{\mathrm{~d} N} & =\frac{2}{(h-1)^{2}} H\left(h, h\left(\zeta_{\max }\right), h\left(\zeta_{\min }\right)\right), \\
& =\frac{2 y^{2}}{(y-q)^{2}} H\left(y, \frac{q}{h\left(\zeta_{\min }\right)}, \frac{q}{h\left(\zeta_{\max }\right)}\right) .
\end{aligned}
$$

Using the formula for the product of two distributions (8), the distribution for the quality factor takes the form

$\frac{\mathrm{d} q}{\mathrm{~d} N} \propto \int_{y_{\min }}^{y_{\max }} \frac{\mathrm{d} y}{y(y-q)^{2}}=q^{-2} \phi(q)$

where $\phi(q)$ contains the details of the ranges of values of $\ell / a$ and $\zeta$

$\phi\left(q,(\ell / a)_{\min },(\ell / a)_{\max }, \zeta_{\min }, \zeta_{\max }\right)=\left[\frac{q}{q-y}+\ln \left|\frac{y}{q-y}\right|\right]_{y_{\min }}^{y_{\max }}$,

and with

$y_{\min }=\max \left(\frac{F}{(\ell / a)_{\max }}, \frac{q}{h\left(\zeta_{\min }\right)}\right)$,
$y_{\max }=\min \left(\frac{F}{(\ell / a)_{\min }}, \frac{q}{h\left(\zeta_{\max }\right)}\right)$.

When $\zeta$ is Jeffrey's distributed, we find the same result as above except that the logarithmic terms in $\phi$ are absent. Equation (12) is simplified if we take the reasonable choices $(\ell / a)_{\min }=0$ and $\zeta_{\min }=1$. With $y_{\min }=F /(\ell / a)_{\max }$ and $y_{\max }=q / h\left(\zeta_{\max }\right)$ Eq. (12) reduces to

$$
\begin{aligned}
\phi\left(q,(\ell / a)_{\max }, \zeta_{\max }\right)= & \frac{h\left(\zeta_{\max }\right)}{h\left(\zeta_{\max }\right)-1}-\ln \left|h\left(\zeta_{\max }\right)-1\right| \\
& -\frac{q(\ell / a)_{\max }}{q(\ell / a)_{\max }-F}-\ln \left|\frac{F}{q(\ell / a)_{\max }-F}\right|
\end{aligned}
$$

The distribution is only physical for $\tau / P$ above a lower threshold value, $(\tau / P)_{\min }$, which occurs where $y_{\min }=y_{\max }$, or

$(\tau / P)_{\min }=\frac{F h\left(\zeta_{\max }\right)}{(\ell / a)_{\max }}$ 


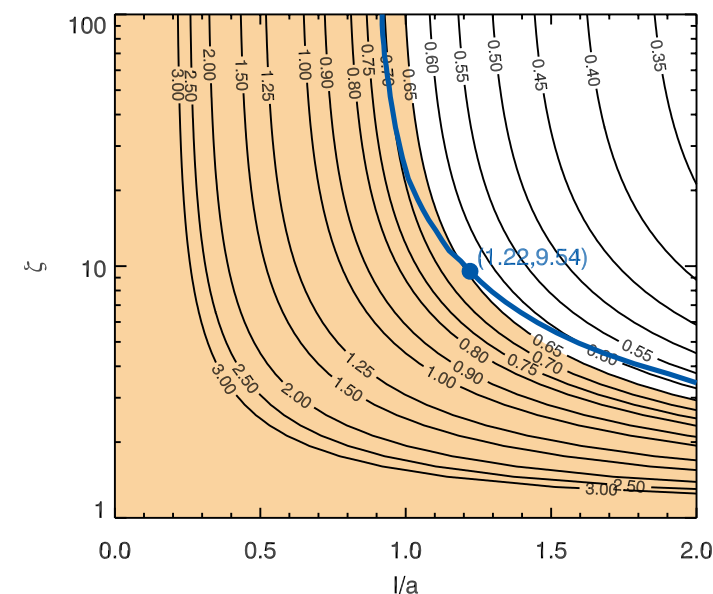

Fig. 7. The allowed range of $\ell / a$ and $\zeta$ for a range of values of $(\tau / P)_{\min }$ as derived using Eq. (15). The ranges exist to the left of each curve as illustrated with the shaded area for $(\tau / P)_{\min }=0.65$. The thick curve represents the value of $\zeta_{\max }$ as a function of $(\ell / a)_{\max }$ that fits best the observed quality factor distribution. A uniform distribution in $\zeta$ has been assumed. The use of a Jeffrey's distribution instead would yield an almost identical curve. The dot indicates the values of $(\ell / a)_{\max }$ and $\zeta_{\max }$ for the fit with the smallest $\chi^{2}$.

It can easily be seen from Eq. (14) that $\phi$ has a root at that value. For $\ell / a$ and $\zeta$ taken from the broadest ranges of $[0,2]$ and $[1, \infty[, \phi$ becomes constant almost everywhere, except near $(\tau / P)_{\min }=F / 2$ where the distribution drops to zero. For that case the distribution scales as $(\tau / P)^{-2}$. The existence of a minimum threshold in the quality factor has been discussed by Goossens et al. (2008) in the context of individual oscillations.

From inspecting the quality factor values of the observations in Fig. 1, one may deduce that except for one all observations have $\tau / P \geq 1$. (There is one observation with a smaller quality factor, but the damping rate has a high uncertainty, Van Doorsselaere et al. 2009.) However, an estimate of $(\tau / P)_{\min }$ based upon the observed lower limit in $\tau / P$ relies on only a few measurements. Instead, we make use of all measurements and fit the observed distribution with Eq. (11) with an arbitrary amplitude and with fixed values of $(\ell / a)_{\min }=0$ and $\zeta_{\min }=1$. As a function of $(\ell / a)_{\max }$, which we determine from the fit the best value of $\zeta_{\max }$. Figure 7 shows this fit as well as the contours of $(\tau / P)_{\min }$ calculated from Eq. (15). It approximately follows the contour of constant $(\tau / P)_{\min }=0.65$. Overall, the best fit is for $\ell / a=1.2$ and $\zeta_{\max }=9.5$ (illustrated in Fig. 5). Then, using Eq. (15) and the value of $(\tau / P)_{\min }=0.65$, we find the constraints

$$
\begin{aligned}
2.9 & \leq \zeta_{\max }=\frac{(\ell / a)_{\max }+0.98}{(\ell / a)_{\max }-0.98}<\infty \\
0.98 & \leq(\ell / a)_{\max } \leq 2
\end{aligned}
$$

For $(\ell / a)_{\max }=2, \zeta$ is constrained to lie in the interval $[1,2.9]$. This result is consistent with what was found in the previous section. Figure 7 shows that loops with simultaneously high values of $\ell / a$ and $\zeta$, i.e. smooth and large contrasted loops, are not consistent with the observations.

\section{Statistics of transverse waves seen by CoMP}

We now turn our attention to the propagating transverse waves observed by CoMP (Tomczyk et al. 2007; McIntosh et al. 2008), and also by SDO/AIA (McIntosh et al. 2011). We interpret

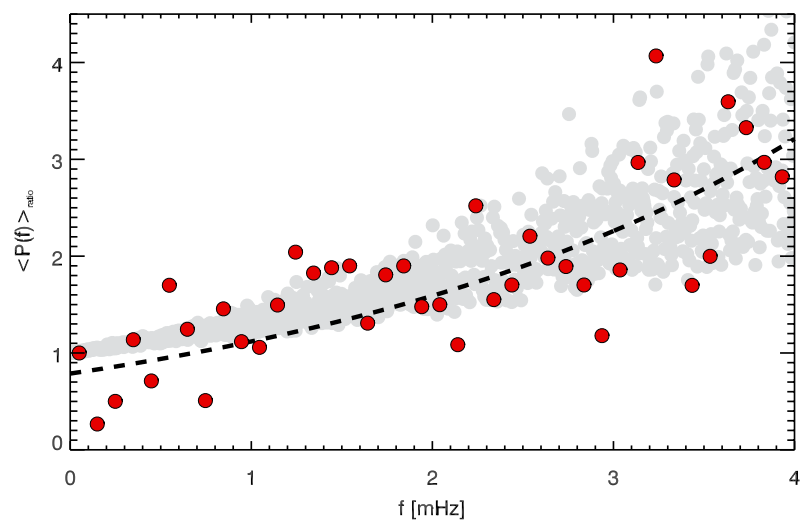

Fig. 8. The CoMP power ratio of downward propagating to upward propagating waves versus the frequency (as taken from Verth et al. 2010) are displayed with red circles. A realisation from 1000 samples from the statistical distributions for $V_{\mathrm{A}, \mathrm{i}} \in[800,1000] \mathrm{km} \mathrm{s}^{-1}, \ell / a \in$ $[0.5,1], \zeta \in[2.5,4]$ are shown as grey circles. The best fit to the observations, Eq. (21), is shown as the dashed line.

the spatial damping as attenuation due to resonant absorption (Verth et al. 2010; Terradas et al. 2010). As in the previous section, we want to perform seismology on the CoMP data in a statistical sense by fitting the data using statistical distributions for the loop cross-sectional equilibrium parameters, i.e. the density contrast $\zeta$, the thickness of the smooth layer $\ell / a$, and the internal Alfvén transit time $\tau_{\mathrm{A}, \mathrm{i}}$.

The data we study is displayed in Fig. 2 of Verth et al. (2010) (also in Fig. 8). They show the ratio of downward propagating wave power (measured as Doppler shifts) with the upward propagating wave power versus the wave frequency. The data is obtained from the $k-\omega$ diagram for CoMP Doppler shifts (McIntosh et al. 2008). In the work of Verth et al. (2010), it is explained that the frequency-dependent power ratio can easily be explained by resonant absorption. In contrast to the previous section with the standing transverse oscillations, the damping for these driven waves is acting as the waves travel along the loop, rather than being a temporal damping of the standing transverse waves.

The waves travel at the phase speed, $v_{\mathrm{ph}}$, which equals the kink speed (Edwin \& Roberts 1983) in the thin loop limit. In the zero plasma- $\beta$ limit, $v_{\mathrm{ph}}$ is thus given by

$v_{\mathrm{ph}}=B \sqrt{\frac{2}{\mu_{\mathrm{o}}\left(\rho_{\mathrm{i}}+\rho_{\mathrm{e}}\right)}}=V_{\mathrm{A}, \mathrm{i}} \sqrt{\frac{2 \zeta}{\zeta+1}}$,

where we have defined $V_{\mathrm{A}, \mathrm{i}}$ as the loop Alfvén speed. Using Eq. (17), we can now compute the frequency dependence of the power ratio (using Eq. (7) in Verth et al. 2010):

$\langle P(f)\rangle_{\text {ratio }}=\frac{P_{\text {out }}(f)}{P_{\text {in }}(f)} \exp (\mathbb{T} f)$,

with

$\mathbb{T}\left(\ell / a, \zeta, V_{\mathrm{A}, \mathrm{i}}\right)=\frac{2 L}{v_{\mathrm{ph}} \xi_{\mathrm{E}}}=\frac{\sqrt{2} L}{F} \frac{1}{V_{\mathrm{A}, \mathrm{i}}}(\ell / a) \frac{\zeta-1}{\sqrt{\zeta(\zeta+1)}}$,

where the previously computed expression for the phase speed $v_{\mathrm{ph}}$ has been used, and the expression for $\xi_{\mathrm{E}}$ from Eq. (2) is inserted. In the remainder of the manuscript we take the measurement length $L=250 \mathrm{Mm}$ from Tomczyk \& McIntosh (2009). The factor $\mathbb{T}$ controls the rate of change of the power 
ratio as a function of frequency. It is a function of the loop parameters $\ell / a, \zeta$ and $V_{\mathrm{A}, \mathrm{i}}$, which are assumed to be fundamental properties of the observed coronal loops. We wish to constrain the values of these variables through statistical means, only for the specific loops. The value of these variables may differ for other loops (especially $V_{\mathrm{A}, \mathrm{i}}$ ) and an over-arching statistical study would have to be conducted using many (currently non-existing) CoMP-like observations to explore statistical constraints on $\ell / a$ and $\zeta$ for all loops, as been done in Sect. 2. We consider the three variables as independent statistical variables, and each of them has a uniform distribution. To the uniform distributions for $\ell / a$ and $\zeta$, described in Eq. (4), we add a third uniform distribution for $V_{\mathrm{A}, \mathrm{i}}$ :

$$
\frac{d V_{\mathrm{A}, \mathrm{i}}}{d N}=H\left(V_{\mathrm{A}, \mathrm{i}}, V_{\mathrm{A}, \mathrm{i}, \min }, V_{\mathrm{A}, \mathrm{i}, \max }\right) .
$$

With these variables, we can calculate the phase speed and power ratio of the transverse waves using Eqs. (17) and (18). To obtain Fig. 8, we generate 1000 different loops, and multiply by a linear function in the frequency domain $f$ to guarantee an even spread across the spectrum. For the sake of simplicity, we take the upward/downward ratio $P_{\text {out }} / P_{\text {in }}=1$, even though Verth et al. (2010) found $P_{\text {out }} / P_{\text {in }}=0.91$ from a fit to the data. A good fit between the data and the statistical loops is obtained for $\ell / a \in$ $[0.5,1], \zeta \in[2.5,4]$ and $V_{\mathrm{A}, \mathrm{i}} \in[800,1000] \mathrm{km} \mathrm{s}^{-1}$. These fitting parameters are not the only ones to yield good results since other combinations may yield similarly good results.

In Fig. 8, it can be observed that the spread of the statistical points increases with increasing frequency. Also, when $f \rightarrow 0$, the spread disappears and the power ratio becomes exactly unity. This is a consequence of the exponential damping by resonant absorption. Indeed, when taking the limit $f \rightarrow 0$ in Eq. (19), the argument goes exactly to 0 . The increasing spread with increasing frequency can also be understood in similar terms. For the sake of simplicity, we assume that $\mathbb{T} \sim N\left(\mu, \sigma^{2}\right)$ has a Gaussian distribution with mean $\mu$ and spread $\sigma$. From elementary statistics, we learn that $\mathbb{T} f \sim N\left(f \mu, f^{2} \sigma^{2}\right)$, resulting in a wider spread for higher frequencies. Also, the mean will increase linearly with $f$, which is observed in Fig. 8 as well.

In the end, only the statistical properties of $\mathbb{T}$ determine the fitting with the observed data points. Indeed, one may increase the spread in $V_{\mathrm{A}, \mathrm{i}}$ by reducing the spread of $\ell / a$ while keeping the average of their product constant. Likewise, the average Alfvén transit time can be decreased by increasing $\ell / a$. Also (as can be seen in Fig. 6), when $\zeta$ is increased, the resulting $\mathbb{T}$ is naturally distributed more narrowly, allowing for a wider spread in $V_{\mathrm{A}, \mathrm{i}}$.

We now take a more statistically rigorous approach. To gain insight into the distribution of $\mathbb{T}$, we calculate the regression of the logarithm of Eq. (18), and we find that

$$
\langle P(f)\rangle_{\text {ratio }}=b \mathrm{e}^{\mathbb{T} f}, \ln b=-0.24 \pm 0.17, \mathbb{T}=350 \pm 70 \mathrm{~s},
$$

The uncertainties are calculated using the vertical spread of the residues $\left(\sigma_{P-P_{\text {fit }}}=0.54\right)$ as an error estimate on the initial measurements and using the method as detailed in Chapter 15.2 of Press et al. (2007). This produces a mean $\mu_{\mathbb{T}}=350 \mathrm{~s}$, and standard deviation $\sigma_{\mathbb{T}}^{2}=4900 \mathrm{~s}^{2}$ of the distribution $\mathbb{T}$. From $\ln b=$ -0.24 , we estimate $P_{\text {out }} / P_{\text {in }}=0.79$. Verth et al. (2010) finds a higher value of $P_{\text {out }} / P_{\text {in }}=0.91$, but since this lies within one sigma of our value, there is statistically no discrepancy.

We now consider $\mathbb{T}$ as a statistical distribution, as the product of the independent statistical variables $V_{\mathrm{A}, \mathrm{i}}, \ell / a$ and $g(\zeta)=$ $(\zeta-1) / \sqrt{\zeta(\zeta+1)}$. As in Sect. 2 , an analytical distribution could be calculated but because $\mathbb{T}$ depends on three statistical variables and the dependency on $\zeta$ is more elaborate, this becomes impractical. Instead, we aim to constrain the independent statistical variables using the fit. Since we assume that $V_{\mathrm{A}, \mathrm{i}}, \ell / a$, and $g(\zeta)$ are independent, it is possible to write

$$
\begin{aligned}
\frac{\sqrt{2}}{\pi L} \mu_{\mathbb{T}}= & \mu_{1 / V_{\mathrm{A}, \mathrm{i}}} \mu_{\ell / a} \mu_{g(\zeta)}, \\
\frac{2}{\pi^{2} L^{2}} \sigma_{\mathbb{T}}^{2}= & \left(\mu_{1 / V_{\mathrm{A}, \mathrm{i}}}^{2}+\sigma_{\tau_{\mathrm{A}, \mathrm{i}}}^{2}\right)\left(\mu_{\ell / a}^{2}+\sigma_{\ell / a}^{2}\right)\left(\mu_{g(\zeta)}^{2}+\sigma_{g(\zeta)}^{2}\right) \\
& -\mu_{1 / V_{\mathrm{A}, \mathrm{i}}}^{2} \mu_{\ell / a}^{2} \mu_{g(\zeta)}^{2} \\
= & \sigma_{1 / V_{\mathrm{A}, \mathrm{i}}}^{2} \sigma_{\ell / a}^{2} \sigma_{g(\zeta)}^{2}+\mu_{1 / V_{\mathrm{A}, \mathrm{i}}}^{2} \sigma_{\ell / a}^{2} \sigma_{g(\zeta)}^{2}+\sigma_{1 / V_{\mathrm{A}, \mathrm{i}}}^{2} \mu_{\ell / a}^{2} \sigma_{g(\zeta)}^{2} \\
& +\sigma_{1 / V_{\mathrm{A}, \mathrm{i}}}^{2} \sigma_{\ell / a}^{2} \mu_{g(\zeta)}^{2}+\mu_{1 / V_{\mathrm{A}, \mathrm{i}}}^{2} \mu_{\ell / a}^{2} \sigma_{g(\zeta)}^{2}+\mu_{1 / V_{\mathrm{A}, \mathrm{i}}}^{2} \sigma_{\ell / a}^{2} \mu_{g(\zeta)}^{2} \\
& +\sigma_{1 / V_{\mathrm{A}, \mathrm{i}}}^{2} \mu_{\ell / a}^{2} \mu_{g(\zeta)}^{2} .
\end{aligned}
$$

All the terms on the right-hand side of Eq. (23) are positive. By considering only the last term and dividing Eq. (23) by the square of Eq. (22), we find

$0.04=\frac{4900}{350^{2}}=\left(\frac{\sigma_{\mathbb{T}}}{\mu_{\mathbb{T}}}\right)^{2}>\left(\frac{\sigma_{1 / V_{\mathrm{A}, \mathrm{i}}}}{\mu_{1 / V_{\mathrm{A}, \mathrm{i}}}}\right)^{2}$,

because positive terms have been neglected on the right-hand side of Eq. (23). Considering only the second and third-to-last term, we can derive

$\left(\frac{\sigma_{\ell / a}}{\mu_{\ell / a}}\right)^{2}<0.04,\left(\frac{\sigma_{g(\zeta)}}{\mu_{g(\zeta)}}\right)^{2}<0.04$

but also

$\left(\frac{\sigma_{1 / V_{\mathrm{A}, \mathrm{i}}}}{\mu_{1 / V_{\mathrm{A}, \mathrm{i}}}}\right)^{2}+\left(\frac{\sigma_{\ell / a}}{\mu_{\ell / a}}\right)^{2}+\left(\frac{\sigma_{g(\zeta)}}{\mu_{g(\zeta)}}\right)^{2}<0.04$

These equations prove that the first four terms in Eq. (23) can be neglected compared with the last three terms.

The relations (24)-(25) contain information on the statistical distribution of $V_{\mathrm{A}, \mathrm{i}}, \ell / a$ and $\zeta$. As we did in the forward modelling, we consider $\ell / a$ as uniformly distributed $H\left(\ell / a,(\ell / a)_{\min },(\ell / a)_{\max }\right)$. From statistics, we know that for this distribution the mean and variance are equal to $\mu_{\ell / a}=\left((\ell / a)_{\min }+\right.$ $\left.(\ell / a)_{\max }\right) / 2$ and $\sigma_{\ell / a}^{2}=\left((\ell / a)_{\max }-(\ell / a)_{\min }\right)^{2} / 12$, respectively. Using Eq. (24), we find

$\left(\frac{\sigma_{\ell / a}}{\mu_{\ell / a}}\right)^{2}=\frac{1}{3}\left(\frac{(\ell / a)_{\max }-(\ell / a)_{\min }}{(\ell / a)_{\min }+(\ell / a)_{\max }}\right)^{2}<0.04$

or using $(\ell / a)_{\max }>(\ell / a)_{\min }$, then

$$
\begin{aligned}
\frac{(\ell / a)_{\max }-(\ell / a)_{\min }}{(\ell / a)_{\min }+(\ell / a)_{\max }} & <\sqrt{0.12} \Rightarrow \\
(\ell / a)_{\max } & <\frac{1+\sqrt{0.12}}{1-\sqrt{0.12}}(\ell / a)_{\min }=2.06(\ell / a)_{\min } .
\end{aligned}
$$

This leaves a rather narrow range for $\ell / a$. If it is expected from physical reasons or loop modelling that the inhomogeneity layer covers the whole loop, $(\ell / a)_{\max }=2$, then the lower boundary for that length scale is $(\ell / a)_{\min }>0.97$.

Also assuming a homogeneous distribution of $V_{\mathrm{A}, \mathrm{i}}$, we can calculate the mean $\mu_{1 / V_{\mathrm{A}, \mathrm{i}}}$ (introducing the notation $\Delta V_{\mathrm{A}}=$ $\left.V_{\mathrm{A}, \mathrm{i}, \max }-V_{\mathrm{A}, \mathrm{i}, \min }\right)$ as

$\mu_{1 / V_{\mathrm{A}, \mathrm{i}}}=E\left[\frac{1}{V_{\mathrm{A}, \mathrm{i}}}\right]=\frac{1}{\Delta V_{\mathrm{A}}} \int_{V_{\mathrm{A}, \mathrm{i}, \min }}^{V_{\mathrm{A}, \mathrm{i}, \max }} \frac{\mathrm{d} V_{\mathrm{A}}}{V_{\mathrm{A}}}=\frac{1}{\Delta V_{\mathrm{A}}} \ln \left|\frac{V_{\mathrm{A}, \mathrm{i}, \max }}{V_{\mathrm{A}, \mathrm{i}, \min }}\right|$, 


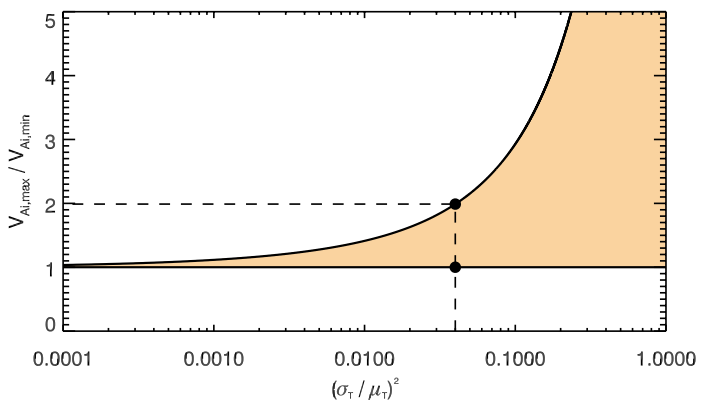

Fig. 9. Uncertainty interval of $V_{\mathrm{A}, \mathrm{i}, \max } / V_{\mathrm{A}, \mathrm{i}, \min }$ as a function of the relative variance $\left(\sigma_{\mathbb{T}} / \mu_{\mathbb{T}}\right)^{2}$, derived from Eq. (31) The interval given by Eq. (32) for $\left(\sigma_{\mathbb{T}} / \mu_{\mathbb{T}}\right)^{2}=0.04$ is indicated.

where $E[$.$] is the expected value of the argument function. The$ variance is computed as

$\sigma_{1 / V_{\mathrm{A}, \mathrm{i}}}^{2}=E\left[\frac{1}{V_{\mathrm{A}, \mathrm{i}}^{2}}\right]-E\left[\frac{1}{V_{\mathrm{A}, \mathrm{i}}}\right]^{2}=\frac{1}{V_{\mathrm{A}, \mathrm{i}, \min } V_{\mathrm{A}, \mathrm{i}, \max }}-\mu_{1 / V_{\mathrm{A}, \mathrm{i}}}^{2}$.

Thus, we find the condition for $V_{\mathrm{A}, \mathrm{i}, \min }$ and $V_{\mathrm{A}, \mathrm{i}, \max }$ :

$0.04>\left(\frac{\sigma_{1 / V_{\mathrm{A}, \mathrm{i}}}}{\mu_{1 / V_{\mathrm{A}, \mathrm{i}}}}\right)^{2}=\frac{\left(\Delta V_{\mathrm{A}}\right)^{2}}{V_{\mathrm{A}, \mathrm{i}, \min } V_{\mathrm{A}, \mathrm{i}, \max }} \frac{1}{\ln ^{2}\left|\frac{V_{\mathrm{A}, \mathrm{i}, \max }}{V_{\mathrm{A}, \mathrm{i}, \min }}\right|}-1$.

This transcendental equation can be solved numerically for $V_{\mathrm{A}, \mathrm{i}, \max } / V_{\mathrm{A}, \mathrm{i}, \min }$, and the result is shown in Fig. 9. For $\sigma_{\mathbb{T}}^{2} / \mu_{\mathrm{T}}^{2}=$ 0.04 , we find

$1<\frac{V_{\mathrm{A}, \mathrm{i}, \max }}{V_{\mathrm{A}, \mathrm{i}, \min }}<1.9885$,

which shows that the loop Alfvén speed is constrained even more narrowly than $\ell / a$.

Interestingly, Tomczyk \& McIntosh (2009) found a phase speed of $600 \mathrm{~km} \mathrm{~s}^{-1}$ for the propagating transverse waves. In our current model (i.e. an overdense loop experiencing resonant damping of the waves), this value is an upper limit for the Alfvén speed in the loop: $V_{\mathrm{A}, \mathrm{i}, \max }=600 \mathrm{~km} \mathrm{~s}^{-1}$. This leads to a minimum value for the Alfvén speed of $V_{\mathrm{A}, \mathrm{i}, \mathrm{min}}=300 \mathrm{~km} \mathrm{~s}^{-1}$, in order to still be able to explain the narrow spread in the observed power ratio. Such low values are conceivable since the magnetic field strength in a loop arcade is expected to decrease with loop length. Alfvén speeds in the range of $300-400 \mathrm{~km} \mathrm{~s}^{-1}$ have been reported for loops with lengths over $650 \mathrm{Mm}$ (Verwichte et al. 2010).

To find estimates for the distribution of parameter $\zeta$, more effort has to be made, because it occurs through the function $g(\zeta)$. As before we assume that $\zeta$ is uniformly distributed $H\left(\zeta, \zeta_{\min }, \zeta_{\max }\right)$. The mean and variance of $g(\zeta)$ can be calculated analytically (see Appendix A). Fixing $\zeta_{\min }$, we can compute the maximally allowed $\zeta_{\max }$ to satisfy Eq. (25). The results of this computation are shown in Fig. 10 where contours of constant values of $\left(\sigma_{g(\zeta)} / \mu_{g(\zeta)}\right)^{2}$ are shown in the parameter space $\zeta_{\min }-\zeta_{\max }$. For $\left(\sigma_{\mathbb{T}} / \mu_{\mathbb{T}}\right)^{2}=0.04$, the valid range of values of $\zeta_{\min }$ and $\zeta_{\max }$ that are consistent with the observations are indicated. The smaller the error in $\mathbb{T}$, the smaller the admissible region becomes (i.e. contours for lower ratios are more to the right). For all values of the error and $\zeta_{\min }, \zeta_{\max } \rightarrow \infty$ is not excluded, and thus $\zeta$ is never constrained. However, realistically, we can assume a reasonable finite upper limit for $\zeta$ of order 10 (e.g. from loop physics it is known that the density contrast $\zeta \leq 10$ ). Then we see that $\zeta_{\max }$ can become even

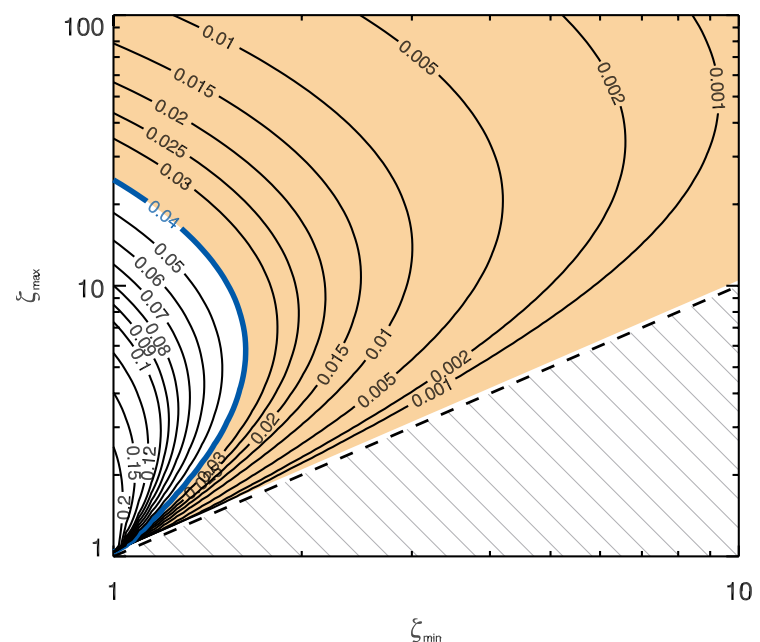

Fig. 10. Contours of constant values of $\left(\sigma_{g(\zeta)} / \mu_{g(\zeta)}\right)^{2}$ as a function of $\zeta_{\min }$ and $\zeta_{\max }$. The dashed line is the bisector and the hatched region indicates unphysical values of $\zeta_{\max }$. The region in parameter space where $\left(\sigma_{g(\zeta)} / \mu_{g(\zeta)}\right)^{2}<0.04$ is shaded.

more constrained for low values of $\zeta_{\min }$ as long as the error in $\mathbb{T}$ is small enough that the contour of this error remains above this upper limit on the left axis $\zeta_{\min }=1$ (e.g. for $\zeta \leq 10$, if $\left(\sigma_{\mathbb{T}} / \mu_{\mathbb{T}}\right)^{2}<0.08$ a much stronger upper limit for $\zeta$ may be found). For $\left(\sigma_{\mathbb{T}} / \mu_{\mathbb{T}}\right)^{2}=0.04$ there is a restricted range of $\zeta$ for $\zeta_{\min }<1.63$. $\zeta_{\min }=1.63$ is the maximal extent of the contour because of the asymptotic behaviour of $g(\zeta)$ (see Fig. 6). If $\zeta_{\min }$ is increased beyond this value, no $\zeta_{\max }$ can be excluded, and no statements can be made on the statistical distribution of $\zeta$.

The external Alfvén speed, $V_{\mathrm{A}, \mathrm{e}}$, is constrained to be within the interval

$\max \left\{v_{\mathrm{ph}}, \sqrt{\zeta_{\min }} V_{\mathrm{A}, \mathrm{i}, \min }\right\}<V_{\mathrm{A}, \mathrm{e}}<\sqrt{\zeta_{\max }} V_{\mathrm{A}, \mathrm{i}, \max }$.

For a fixed value of $\sigma_{\mathbb{T}} / \mu_{\mathbb{T}}$, using Eq. (31) and taking $v_{\mathrm{ph}}=$ $V_{\mathrm{A}, \mathrm{i}, \text { min }}$, the ratio $\left(V_{\mathrm{A}, \mathrm{e}} / V_{\mathrm{A}, \mathrm{i}, \mathrm{min}}\right)^{2}$ is determined by $\zeta_{\min }$, i.e.

$\max \left\{\left(\frac{V_{\mathrm{A}, \mathrm{i}, \max }}{V_{\mathrm{A}, \mathrm{i}, \min }}\right)^{2}, \zeta_{\min }\right\}<\left(\frac{V_{\mathrm{A}, \mathrm{e}}}{V_{\mathrm{A}, \mathrm{i}, \min }}\right)^{2}<\left(\frac{V_{\mathrm{A}, \mathrm{i}, \max }}{V_{\mathrm{A}, \mathrm{i}, \min }}\right)_{\max }^{2} \zeta_{\max }$.

We may employ again Fig. 10 to determine the interval of admissible values. For $\left(\sigma_{\mathbb{T}} / \mu_{\mathbb{T}}\right)^{2}=0.04, \max \left(V_{\mathrm{A}, \mathrm{i}, \max } / V_{\mathrm{A}, \mathrm{i}, \min }\right)=$ 1.9885 and $V_{\mathrm{A}, \mathrm{i}, \max }=600 \mathrm{~km} \mathrm{~s}^{-1}$, we find the following constraints for $V_{\mathrm{A}, \mathrm{e}}$. For $\zeta_{\min }=1, V_{\mathrm{A}, \mathrm{e}}$ is constrained to be exactly equal to $V_{\mathrm{A}, \mathrm{i}, \max }$. For increasing $\zeta_{\min }$, the upper bound increases until for $\zeta_{\min }=1.63$ the interval becomes $600 \mathrm{~km} \mathrm{~s}^{-1}<V_{\mathrm{A}, \mathrm{e}}<$ $800 \mathrm{~km} \mathrm{~s}^{-1}$. For $1.63<\zeta_{\min } \leq 3.95, V_{\mathrm{A}, \mathrm{e}}$ is only constrained to be larger than $600 \mathrm{~km} \mathrm{~s}^{-1}$. For $\zeta_{\min }>3.95$, the lower bound needs to be larger than $\sqrt{\zeta_{\min }} 300 \mathrm{~km} \mathrm{~s}^{-1}$.

\section{Conclusions}

We have explored how coronal loop cross-sectional characteristics, such as density contrast and transition layer width, can be seismologically constrained using observations of transverse waves and oscillations through a statistical approach.

We investigated TLOs, the majority of which have been observed by TRACE and SDO. First, we produced many observed realisations of TLOs periods and damping times from sampled values of $\ell / a$ and $\zeta$ through forward-modelling. The index of the power-law fit follows a Gaussian distribution centred on an index value of one. The uncertainty (Gaussian width) of the index 
strongly depends on the range of periods used. For a range of periods between 140 and $440 \mathrm{~s}$, as seen by Aschwanden et al. (2002), the uncertainty becomes as much as 0.5 . But for a period between 50 and $3600 \mathrm{~s}$, the uncertainty is only 0.14 . We have demonstrated that the observed period-damping time scaling law does provide information about the physical damping mechanism, provided observations of TLOs are collected from a range of periods as wide as possible and provided a comparison with theory is performed in a statistical sense. Important is that we have assumed that the distributions of these two parameters from which we sample is the same for all periods (hence also loop length). We did not take the possibility of different classes of coronal loops present within the observations into account. We are confident that this is a reasonable assumption because most reported TLOs come from similarly sized active region loops. Without this assumption the observed TLOs would somehow have widely different cross-sectional structuring that also depends on period, at which point almost any power law may be expected (Arregui et al. 2008).

With the full range of values for $\ell / a$ and $\zeta_{\min }=1$, we find the best fit as a function of the one remaining free parameter, $\zeta_{\max }$, equal to 3 . However, the error in both the observational and the forward-modelling of the value of $\alpha$ is large. Instead, to have more statistics, we have reduced the problem to a onedimensional distribution of the quality factor $\tau / P$. For a given distribution of $\ell / a$ and $\zeta$ we calculated the shape of the quality factor distribution. As already noted by Goossens et al. (2008), $\tau / P$ has a minimum value determined by $(\ell / a)_{\max }$ and $\zeta_{\max }$. Fitting the observed quality-factor distribution of TLOs showed that loops with high values of $\ell / a$ and $\zeta$ are not consistent with the observations. It is important to note that such loops would support transverse oscillations with a poor quality factor, whose transverse oscillations for typical displacement amplitudes and periods would be difficult to detect. There is an observer bias against studying such loops. Therefore, instead of relying only on the lower limit of observed quality factor to constrain $\ell / a$ and $\zeta$, we determined it by fitting the distribution curve to all observations, and find $(\tau / P)_{\min }=0.65$. From the resulting relation between $\zeta_{\max }$ and $(\ell / a)_{\max }$, we see that the density contrast is only constrained if $(\ell / a)_{\max }$ is greater than 0.98 , and that $\zeta_{\max }$ decreases as a function of $(\ell / a)_{\max }$ until a value of approximately 3 for $(\ell / a)_{\max }=2$. The latter value is broadly consistent with the scaling law fitting. Loops with simultaneously high values of $\ell / a$ and $\zeta$, i.e. smooth and highly contrasted loops, are inconsistent with the observations. In interpreting these results we need to bear in mind that the analytical resonant absorption equation is, strictly speaking, only valid for low values of $\ell / a$. This study could be repeated using numerical solutions of the resonant absorption problem, which allows for an arbitrary transition-layer thickness (e.g. Van Doorsselaere et al. 2004).

For the CoMP running transverse waves, we reproduced the observed power-ratio dependency with the frequency, using uniform distributions for $\ell / a, V_{\mathrm{A}, \mathrm{i}}$, and $\zeta$ (as for the TLOs). In the forward modelling, it became obvious that relatively narrow ranges had to be used to fit the observed spread on the data points. Taking a more statistically rigorous approach, we studied the mean and the spread on the parameter $\mathbb{T}$. This parameter contains all the statistical information of the loop. From the fitted values and spread of $\mathbb{T}$, it was possible to constrain the distributions of $\ell / a, V_{\mathrm{A}, \mathrm{i}}$, and $\zeta$. It was found that the ratio of upper and lower bounds on the uniform distribution of $\ell / a$ and $\tau_{\mathrm{A}, \mathrm{i}}$ should be less than 2.06 , to comply with the statistical properties of $\mathbb{T}$. Additionally, the distribution for the loop density contrast could be either extremely narrow or wide. Depending on other physical estimates for upper limits of the density contrast, a severe restriction could be found for the density contrast distribution. A similar analysis could not be made for the TLOs because the uncertainty on the fit parameter is too large.

The statistical study of TLOs permits constraints to be determined on the physical characteristics of coronal loops as a whole. One of the main unanswered questions surrounding coronal wave dynamics is the selective (degree of) excitation of loops in active regions. It is possible that the physical mechanisms that excite these oscillations, e.g. shocks, heating, and pressure imbalance, are selective in, for example, direction or distance. But another possible explanation may be formulated from the result of $(\tau / P)_{\min }$. It reveals that some loops may not be seen (reported) to oscillate transversely because they have oscillations with a quality factor that is too small to be detected as an oscillation by an observer. The CoMP study reveals narrow distributions for the statistical parameters, which suggests that all loops observed with CoMP have similar properties. A rather narrow distribution for loop-density contrast is found. Perhaps our result is the consequence of only studying one time series in one active region but it may also reveal a strong constraint on the understanding and modelling of loop physics. Are the oscillating loops a special subset of the large ensemble of coronal loops, or do all loops have e.g. a density contrast in a narrow range?

To decrease the uncertainties we need many more observations of standing and running transverse waves in as wide a range of active regions/temperatures and periods as possible. The characterisation of TLOs is a labour-intensive data-analysis process because of the need for the detection of transverse displacements in images. Systematic and consistent methods of analysis of TLOs are being developed (Verwichte et al. 2009, 2010; White $\&$ Verwichte 2012). A much larger set of observations would allow us to explore the existence of different sub-classes of coronal loops, possibly linked to specific regions. Also, it would require further coronal loop modelling and observational studies of nonoscillating loops.

Acknowledgements. E.V. acknowledges financial support from the UK Science and Technology Facilities Council (STFC) on the CFSA Rolling Grant and the SF fellowship of the KU Leuven Research Council with number SF/12/004, T.V.D. and P.A. have received funding from the Odysseus programme of the FWO-Vlaanderen. T.V.D. also acknowledges funding from the EU's Framework Programme 7 as an ERG with grant number 276808. R.S.W. would like to acknowledge the support of an STFC Ph.D. studentship.

\section{Appendix A: Mean and variance of $g(\zeta)$}

For $\zeta$ uniformly distributed $H\left(\zeta, \zeta_{\min }, \zeta_{\max }\right)$, the mean and variance of $g(\zeta)$ can be calculated analytically. The mean is found (introducing the notation $\Delta \zeta=\zeta_{\max }-\zeta_{\min }$ ) to be

$$
\begin{aligned}
\mu_{g(\zeta)} & =\frac{1}{\Delta \zeta} \int_{\zeta_{\min }}^{\zeta_{\max }} g(\zeta) \mathrm{d} \zeta, \\
& =\frac{1}{\Delta \zeta} \int_{\zeta_{\min }}^{\zeta_{\max }} \frac{\zeta-1}{\sqrt{\zeta(\zeta+1)}} \mathrm{d} \zeta, \\
& =\frac{1}{\Delta \zeta}[\sqrt{\zeta(\zeta+1)}-3 \operatorname{arcsinh}(\sqrt{\zeta})]_{\zeta_{\min }}^{\zeta_{\max }}
\end{aligned}
$$

The variance can be calculated through

$$
\sigma_{g(\zeta)}^{2}=E\left[g^{2}\right]-(E[g])^{2}=E\left[g^{2}\right]-\mu_{g(\zeta)}^{2},
$$


where $E[$.$] stands for the expected value of the argument func-$ tion. For $g(\zeta)$, we find

$$
\begin{aligned}
E\left[g^{2}\right] & =\frac{1}{\Delta \zeta} \int_{\zeta_{\min }}^{\zeta_{\max }} g^{2}(\zeta) \mathrm{d} \zeta, \\
& =\frac{1}{\Delta \zeta} \int_{\zeta_{\min }}^{\zeta_{\max }} \frac{(\zeta-1)^{2}}{\zeta(\zeta+1)} \mathrm{d} \zeta, \\
& =\frac{1}{\Delta \zeta}[\zeta+\ln (\zeta)-4 \ln (\zeta+1)]_{\zeta_{\min }}^{\zeta_{\max }},
\end{aligned}
$$

which is combined with Eq. (A.1) to compute Eq. (A.2).

\section{References}

Arregui, I., \& Asensio Ramos, A. 2011, ApJ, 740, 44

Arregui, I., Andries, J., Van Doorsselaere, T., Goossens, M., \& Poedts, S. 2007, A\&A, 463, 333

Arregui, I., Ballester, J. L., \& Goossens, M. 2008, ApJ, 676, L77

Aschwanden, M. J., Fletcher, L., Schrijver, C. J., \& Alexander, D. 1999, ApJ, 520,880

Aschwanden, M. J., de Pontieu, B., Schrijver, C. J., \& Title, A. M. 2002, Sol. Phys., 206, 99

Aschwanden, M. J., Nightingale, R. W., Andries, J., Goossens, M., \& Van Doorsselaere, T. 2003, ApJ, 598, 1375

De Moortel, I., \& Brady, C. S. 2007, ApJ, 664, 1210

Edwin, P. M., \& Roberts, B. 1983, Sol. Phys., 88, 179

Goossens, M., Hollweg, J. V., \& Sakurai, T. 1992, Sol. Phys., 138, 233

Goossens, M., Andries, J., \& Aschwanden, M. J. 2002, A\&A, 394, L39

Goossens, M., Arregui, I., Ballester, J. L., \& Wang, T. J. 2008, A\&A, 484, 851

Goossens, M., Andries, J., Soler, R., et al. 2012, ApJ, 753, 111

Handy, B. N., Acton, L. W., Kankelborg, C. C., et al. 1999, Sol. Phys., 187, 229

Hollweg, J. V., \& Yang, G. 1988, J. Geophys. Res., 93, 5423

Hori, K., Ichimoto, K., Sakurai, T., Sano, I., \& Nishino, Y. 2005, ApJ, 618, 1001
Hori, K., Ichimoto, K., \& Sakurai, T. 2007, in New Solar Physics with Solar-B Mission, eds. K. Shibata, S. Nagata, \& T. Sakurai, ASP Conf. Ser., 369, 213 Howard, R. A., Moses, J. D., Vourlidas, A., et al. 2008, Space Sci. Rev., 136, 67 Ionson, J. A. 1978, ApJ, 226, 650

Lemen, J. R., Title, A. M., Akin, D. J., et al. 2012, Sol. Phys., 275, 17

McIntosh, S. W., de Pontieu, B., \& Tomczyk, S. 2008, Sol. Phys., 252, 321

McIntosh, S. W., de Pontieu, B., Carlsson, M., et al. 2011, Nature, 475, 477

Mrozek, T. 2011, Sol. Phys., 270, 191

Nakariakov, V. M., \& Ofman, L. 2001, A\&A, 372, L53

Nakariakov, V. M., Ofman, L., Deluca, E. E., Roberts, B., \& Davila, J. M. 1999, Science, 285,862

Ofman, L., \& Aschwanden, M. J. 2002, ApJ, 576, L153

Pascoe, D. J., Hood, A. W., de Moortel, I., \& Wright, A. N. 2012, A\&A, 539, A37

Press, W. H., Teukolsky, S. A., Vetterling, W. T., \& Flannery, B. P. 2007, Numerical Recipes: The Art of Scientific Computing

Ruderman, M. S., \& Roberts, B. 2002, ApJ, 577, 475

Schmelz, J. T., Beene, J. E., Nasraoui, K., et al. 2003, ApJ, 599, 604

Terradas, J., Goossens, M., \& Verth, G. 2010, A\&A, 524, A23

Terzo, S., \& Reale, F. 2010, A\&A, 515, A7

Tomczyk, S., \& McIntosh, S. W. 2009, ApJ, 697, 1384

Tomczyk, S., McIntosh, S. W., Keil, S. L., et al. 2007, Science, 317, 1192

Tomczyk, S., Card, G. L., Darnell, T., et al. 2008, Sol. Phys., 247, 411

Van Doorsselaere, T., Andries, J., Poedts, S., \& Goossens, M. 2004, ApJ, 606, 1223

Van Doorsselaere, T., Nakariakov, V. M., \& Verwichte, E. 2007, A\&A, 473, 959

Van Doorsselaere, T., Birtill, D. C. C., \& Evans, G. R. 2009, A\&A, 508, 1485

Verth, G., Terradas, J., \& Goossens, M. 2010, ApJ, 718, L102

Verwichte, E., Nakariakov, V. M., Ofman, L., \& Deluca, E. E. 2004, Sol. Phys., 223, 77

Verwichte, E., Foullon, C., \& Nakariakov, V. M. 2006, A\&A, 452, 615

Verwichte, E., Aschwanden, M. J., Van Doorsselaere, T., Foullon, C., \& Nakariakov, V. M. 2009, ApJ, 698, 397

Verwichte, E., Foullon, C., \& Van Doorsselaere, T. 2010, ApJ, 717, 458

Verwichte, E., Van Doorsselaere, T., White, R., Bacon, A., \& Williams, A. 2012, A\&A

Wang, T. J., \& Solanki, S. K. 2004, A\&A, 421, L33

Wang, T., Ofman, L., Davila, J. M., \& Su, Y. 2012, ApJ, 751, L27

Wentzel, D. G. 1979, ApJ, 227, 319

White, R. S., \& Verwichte, E. 2012, A\&A, 537, A49

White, R. S., Verwichte, E., \& Foullon, C. 2012, A\&A, 545, A129 